\title{
INFLUENCE DE L'INGESTION D'ANTIOXYGÈNES SUR LA COMPOSITION DE CERTAINS TISSUS ET SUR LA STABILITÉ DES GRAISSES DE RESERVE DU PORC ET DU POULET
}

\author{
PAR \\ A.-C. François et Andrée PIHET \\ Avec la collaboration technique de Jacqueline Thevenoux \\ Service de Biochimie et de Nutrition CNRZ, Jouy-en-Josas (S et O).
}

\section{SOMMAIRE}

L'influence de l'ingestion de divers antioxygènes surleur accumulation éventuelle dans le muscle, les graisses de réserve (dorsale et périnéphrétique), le foie et le rein, ainsi que sur la résistance à l'oxydation des graisses de réserve, a été étudiée chez le porc et le poulet.

Les animaux recevaient dans leur ration soit 0,125 p. Ioo de diphényl-p. phénylènediamine (DPPD), soit $O, I$ p. Ioo de butylhydroxyanisol (BHA), de butylhydroxytoluène $\left(\mathrm{BH}^{\prime} \mathrm{T}\right)$, de gallate de dodécyle, de gallate de propyle, ou d'acide nordihydroguaïarétique (NDGA).

Chez le porc, seul le DPPI) est décelable dans les graisses de réserve (taux maximum trouvé : 0,0025 p. I00), et dans le rein (maximum 0,0006 p. IOo). Chez le poulet, le DPPD s'accumule également dans les graisses de réserve, mais la concentration est plus faible $(0,0007 \mathrm{p}$. I0o) que dans la graisse de porc. En revanche, le BH'T est stocké dans la graisse de poulet au taux de 0,006 p. Ioo.

L'étude de la stabilité des graisses de réserve des animaux témoins et des animaux traités confirme le résultat du dosage direct des antioxygènes. En effet, les graisses provenant des porcs ayant ingéré du DPPD et celles des poulets ayant reçu du BHT présentent une période d'induction beaucoup plus longue que celle des graisses d'animaux témoins.

Les conséquences technologiques et hygiéniques de ces observations sont discutées. 
Certains antioxygènes naturels tels que les tocophérols, contenus normalement dans le régime alimentaire des animaux, sont capables d'accroître la résistance à l'oxydation des graisses de réserve. Ce fait a été démontré chez le rat (BARNEs et al. I943; I UNDBERG et al. I944; HANSON et al. I944; BURr, I,UNDBirg et ChIPAUl'́ I946; I,UNDBERG et al. I947), le lapin (MAJOR et WATTS, I948), le pore (CARPENTER et I, Undberg I949; Chipault, I,Undbirg et Burr, I945; Watts, Cunha et Major, r946), le poulet (Mecchi et a1. I956, a b ; HoOD, WHEELER et MeGlanery, I950), le dindon (Mecchi, Pool et Klosis, i953 ; Criddre et MORGAN, I95I).

I es recherches portant sur d'autres antioxygènes et notamment sur les produits de synthèse sont peu nombrenses. Lorsque le présent travail a été entrepris, en I957, l'un des rares travaux publiés portait sur le métabolisme de la diphénỵl-p.-phénylènediamine chez le poulet (I'LDEIKIEWICZ et al. I956). Un peu plus tard une courte étude sur le porc a été publiée (Lynch, Anderson et I.FWrs, I958). Antérieurement BARNEs et al. (I943) avaient montré que l'hydroquinone ingérée, loin de stabiliser les graisses de réserve du rat, provoquait au contraire une accélération de l'oxydation. Or, l'utilisation des antioxygènes en alimentation animale est le corollaire obligatoire de l'incorporation des graisses aux aliments. Ces substances peuvent être également utilisées pour la stabilisation des aliments contenant des graisses oxydables tels que les farines de poisson. On sait en effet, que l'oxydation des graisses alimentaires risque de provoquer, chez les animaux qui les ingèrent, des accidents nutritionnels dont l'origine résulte directement ou indirectement de la présence des produits d'oxydation. Ainsi, afin de prévenir certains de ces accidents tels que l'encéphalomalacie, on incorpore certains antioxygènes à la ration alimentaire des animaux.

I,e présent travail a pour objet d'étudier chez le porc et le poulet quelles sont les conséquences de l'ingestion de divers antioxygènes sur la composition des tissus animaux consommés par l'homme.

\section{MATÉRIEL et MÉTHOUES}

\section{Antioxygènes utilisés — animaux}

Les antioxygènes suivants ont été étudiés : gallate de propyle; gallate de dodécyle; butylhydroxytoluène (BH'T) (ou dibutylparacrésol : $\mathrm{DBPC}$ ) ; acide nordihydroguaiarétique (NDGA); butylhydroxyanisole (BHA) ; diphénylparaphénylènediamine (DPPD). I a dose incorporée aux aliments était de $0, I$ p. Ioo pour tous les produits, sauf pour le DPPD qui était incorporé au taux de 0, I25 p. I00. Ce taux correspond à dix fois 
celui qui est généralement recommandé pour stabiliser les graisses alimentaires.

Pour les porcs, la durée d'administration du régime enrichi d'antioxygènes était de quatre mois environ (poids de 50 à $100 \mathrm{~kg}$ ). Chacun des lots, dont un lot témoin, comprenait 6 animaux. Pour les poulets, l'expérience avait une durée de huit semaines. Chacun des lots comprenait I $_{5}$ oiseaux.

\section{Organes et tissus étudiés}

Chez le porc, le lard dorsal était prélevé dès l'abattage. Les deux couches qui constituent ce tissu étaient séparées et analysées séparément. En outre, on prélevait les graisses périnéphrétiques (panne). Ces tissus gras étaient fondus sous atmosphère de $\mathrm{CO}_{2}$, filtrés et centrifugés. Les déterminations suivantes étaient effectuées sur ces graisses : taux d'antioxygènes, indice d'iode, indice de péroxydes, test de stabilité. Ce dernier test était effectué à l'étuve $60^{\circ} \mathrm{C}$, éclairée, selon une méthode recommandée par Desnukide et par WOLFF. Le muscle semi-tendineux, le foie et le rein faisaient également l'objet de prélèvements à des fins d'analyses. Chez le poulet, les graisses de réserve étaient souvent absentes, bien que nous ayons conduit les animaux jusqu'à $\mathbf{~} 6$ semaines, afin d'obtenir un engraissement suffisant. Nous n'avons donc pu étudier les réserves individuelles. Pour obtenir une quantité suffisante de graisses, nous avons dû réunir les prélèvements correspondant à deux ou trois animaux. Pour le tissu musculaire, les muscles pectoraux et les muscles de la patte ont été prélevés.

\section{Dosage}

\section{Graisses}

Les indices d'iode étaient déterminés selon la méthode de Wijs et les indices de péroxydes (Lea) par iodométrie.

\section{Antioxygènes}

La méthode de KaHaN (I954) à $1^{\prime} \alpha-\alpha^{\prime}$ dipyridyl a été utilisée pour doser le NDGA et le BHA. Elle a été également appliquée au dosage des gallates. En effet, 1a ration de chacun des lots ne contenait qu'un seul antioxygène et, en conséquence, le manque de spécificité de la réaction, qui est un obstacle lorsque l'on a affaire à tun mélange, ne présentait pas d'inconvénient. Il est nécessaire de tracer une courbe d'étalonnage pour chacune des séries de dosages mettant en œuvre cette réaction, quel que soit l'antioxygène dosé. Les lectures étaient effectuées au spec- 
trophotomètre à $5 \mathrm{I} 5 \mathrm{~m} \mu$; le temps de réaction était de $30 \mathrm{mn}$ pour le BHA et les gallates, et de $3 \mathrm{mn}$ pour le NDGA. La modification introduite par Anglin, Mahon et Chapman était appliquée pour le dosage du BHT (addition de n-butanol).

Pour le DPPD on préparait le dérivé nitré qui est dosé par colorimétrie (BunNEL, I954). Dans le cas du dosage de cet antioxygène dans les graisses, nous avons dû procéder à une saponification préalable, après avoir vérifié que celle-ci ne modifiait pas la validité du dosage $(5 \mathrm{~g}$ de graisse, $2 \mathrm{~g}$ de $\mathrm{KOH}, 50 \mathrm{ml}$ d'alcool, pendant tune demi-heure à l'ébullition).

L'extraction des antioxygènes à partir des tissus et organes pose certains problèmes. En particulier, le BH'T doit être entraîné par la vapeur surchauffée, selon la méthode de Anglin, Mahon et Chapman (1956). Nous avons cependant trouvé que la température de distillation proposée par ces auteurs ne convenait pas; elle doit être portée à $I 80^{\circ} \mathrm{C}$ afin d'obtenir une récupération quantitative de l'antioxygène.

Dans le cas des tissus gras, ceux-ci sont dissous dans l'éther de pétrole. L'antioxygène (BHA, NDGA, gallates) est réextrait par l'alcool 72 p. roo. Pour le muscle, l'extraction du BHA et des gallates s'effectue à l'aide d'éther de pétrole, au blendor, selon la méthode de HANLEY (I954). Par ailleurs, la présence de gallates à des concentrations extrêmement faibles peut être détectée par addition de quelques gouttes d'ammoniaque concentré (WENGER, I954).

\section{RÉSULTATS}

\section{Validité des méthodes de dosage}

Des tests de surcharge ont été effectués avec les différents antioxygènes étudiés, a fin de déterminer la limite inférieure de sensibilité, ainsi que la validité du dosage. Ces surcharges ont été effectuées sur le tissu gras, sur le muscle, sur le foie et sur le rein. Les concentrations étudiées variaient de $0,00 \mathrm{I}$ p. IOO à $0, \mathrm{I}$ p. IOO.

\section{a) Graisse.}

Les taux de récupération atteignent pour plusieurs antioxygènes des valeurs de 1'ordre de $95 \mathrm{p}$. Ioo, même pour des concentrations aussi faibles que o,OOI p. Ioo.

Toutefois, la récupération est plus faible ( $90 \mathrm{p}$. Ioo environ) pour ce qui concerne le gallate de propyle et le BHT. Dans ce dernier cas, la succession des opérations de dosage, qui comporte notamment un entraînement à la vapeur surchauffée, est vraisemblablement responsable de ce taux relativement faible. En aucun cas, il n'a été possible de 
confirmer les résultats de Anglin, Mahon et Chapman. En appliquant strictement la méthode proposée par ces auteurs, sans modifier la température de distillation, les taux de récupération ne dépassaient pas 50 à 60 p. 100.

\section{b) Muscle et oRganes.}

Les récupérations des surcharges sont également de l'ordre de 95 p. Ioo pour ce qui concerne le muscle. Pour le rein et le foie, certaines difficultés se présentent pour le DPPD et le gallate de propyle. Enfin, nous avons dû renoncer à doser le BHT dans ces tissus au moyen de la méthode de Anglin, Mahon et Chapman. En effet, la vapeur surchauffée entraîne des substances volatiles réductrices qui rendent impossible le dosage spécifique du BHT.

L'application de ces méthodes nous a permis d'obtenir les résultats réunis dans les tableaux I à IV. On a indiqué, pour le porc, les valeurs extrêmes et les valeurs individuelles. Pour le poulet, on trouve les teneurs moyennes et les teneurs correspondant aux graisses provenant de plusieurs animaux, pour la raison déjà mentionnée.

\section{Passage des antioxygènes dans les tissus}

Les méthodes étudiées ont été appliquées aux différents tissus et organes prélevés dans les conditions décrites ci-dessus.

Porc

\section{TABLEAU I}

Teneur en antioxygènes des tissus du porc

\begin{tabular}{|c|c|c|c|c|c|c|c|}
\hline & $\begin{array}{c}\text { Lard } \\
\text { externe }\end{array}$ & $\begin{array}{l}\text { Lard } \\
\text { interne }\end{array}$ & Panne & Muscle & lioie & Rein & $\operatorname{Sang}$ \\
\hline Gallate de uropyle & [1. 100 & P. 100 & P. 100 & l. 100 & 1). 100 & P. 100 & I) 100 \\
\hline Gallate de dodécyle & 0 & 0 & 0 & o & 0 & 0 & 0 \\
\hline BIIA $\ldots \ldots \ldots$ & o & 0 & 0 & o & o & 0 & 0 \\
\hline NDGA ......... & o & 0 & 0 & 0 & o & o & 0 \\
\hline $\mathrm{BH} \mathrm{H}^{\prime} \ldots \ldots \ldots \ldots$ & $\mathrm{o}$ & $\mathrm{O}$ & 0 & 0 & 0 & 0 & 0 \\
\hline DBPC $\ldots \ldots \ldots$ & 0 & o & o & o & o & 0 & 0 \\
\hline DPPD $\ldots \ldots \ldots$ & o d̀ 0,0025 & o à 0,0024 & $\begin{array}{c}0,0012 \text { à } \\
0,0024\end{array}$ & 0 & o & o à 0,0006 & o \\
\hline
\end{tabular}

I Les résultats du tableau I montrent que les antioxygènes qu'il est possible de doser dans de bonnes conditions ne s'accumulent pas dans le tissu adipeux, ni dans le muscle, ni dans le foie, ni dans le rein, ni dans le sang. I'acide gallique a été également recherché, car un essai effectué in-vitro a montré que les gallates sont rapidement hydrolysés par le contenu intestinal du porc. Par exemple, après incubation dans ce milieu, il est possible de retrouver quantitativement le BHA, alors que les gal_ 
lates disparaissent complètement. Or, les tests qualitatifs de l'acide gallique (Fe $\mathrm{Cl}_{3}$ ) appliqués aux tissus gras et aux organes ont toujours été négatifs.

Un seul antioxygène, toutefois, fait exception à cette règle : il s'agit de la diphénylparaphénylènediamine (IPPD).

I,es taux de DPPD trouvés dans le lard et dans la panne sont toutefois faibles en valeur absolue : 0,0025 p. Ioo au maximum, soit $25 \mathrm{mg}$ par kg de graisse. Notons que cette faible concentration suffit néanmoins à communiquer, dans certains cas, à la graisse une faible coloration rose. Nous verrons par ailleurs que cette concentration suffit également à en retarder considérablement l'oxydation.

\section{TABLEAU II}

Teneur de divers tissus du porc en DPPD

Valeurs individuelles

\begin{tabular}{|c|c|c|c|c|c|c|c|}
\hline Porc & $\begin{array}{l}\text { Iard } \\
\text { externe }\end{array}$ & $\begin{array}{c}\text { Iard } \\
\text { interne }\end{array}$ & Panne & Foie & Muscle & Rein & Sang \\
\hline$\Lambda$ & $\begin{array}{l}\text { P. } 100 \\
0,0022\end{array}$ & $\begin{array}{l}\text { 1). } 100 \\
0,0024\end{array}$ & $\begin{array}{l}\text { 1). } 100 \\
0,0024\end{array}$ & p. 100 & p. roo & $\begin{array}{l}\text { P. } 100 \\
0,0006\end{array}$ & p. 100 \\
\hline$B$ & 0 & 0,0014 & $0,00 \mathrm{I} 6$ & 0 & 0 & 0,0006 & 0 \\
\hline C. & 0,0025 & 0,0024 & 0,0023 & 0 & 0 & o & 0 \\
\hline D & 0,00004 & 0,000194 & 0,0012 & 0 & 0 & 0,0005 & 0 \\
\hline $\mathrm{T}$ & 0 & 0 & 0,0023 & 0 & 0 & 0,0005 & 0 \\
\hline $\mathrm{C}$ & 0,0022 & 0,0015 & 0,0024 & 0 & 0 & 0,0006 & 0 \\
\hline
\end{tabular}

I1 existe des variations individuelles dans la quantité d'antioxygènes contenue dans la graisse (tableau II). Ènfin, parmi les autres tissus et organes, le rein est capable de retenir du LPPD; la concentration trouvée est de l'ordre de 0,0005 p. Ioo, alors que ni le muscle, ni le foie, ni le sang ne contiennent de quantités dosables de cet antioxygène.

Poulet.

L'étude des tissus du poulet, résumée dans les tableaux III et IV

TABLEAU III

Teneurs moyennes en antioxygènes des tissus de poulets

\begin{tabular}{|c|c|c|c|}
\hline & Graisse & Muscle & Foie \\
\hline & p. Ioo & p. 100 & p. 100 \\
\hline Gallate de propyle.$\ldots \ldots \ldots \ldots \ldots$ & 0 & 0 & 0 \\
\hline Gallate de dodécyle $\ldots \ldots \ldots \ldots \ldots \ldots$ & 0 & 0 & o \\
\hline BHA $\ldots \ldots \ldots \ldots \ldots \ldots \ldots$ & o & o & 0 \\
\hline $\mathrm{NDGA}$ & 0 & $\circ$ & $\circ$ \\
\hline BIIT $\ldots \ldots \ldots \ldots \ldots \ldots \ldots \ldots \ldots$ & 0,006 & 0 & o \\
\hline DPPD $\ldots \ldots \ldots \ldots \ldots \ldots \ldots \ldots \ldots \ldots$ & 0,0007 & o & o \\
\hline
\end{tabular}


TABLEAU IV

Teneur des divers tissus du poulet en DPPD.

\begin{tabular}{|c|c|c|c|c|}
\hline & & Graisse & Muscle & Foie \\
\hline & & 1. 100 & 1) 100 & P. 100 \\
\hline Groupe 9 (3 poulets) & $\ldots \ldots \ldots \ldots \ldots$ & 0,001 & $\circ$ & 0 \\
\hline Groupe ro (3 poulets) & $\ldots \ldots \ldots \ldots$ & 0,0005 & $\circ$ & 0 \\
\hline Groupe i I (3 poulets) & $\ldots \ldots \ldots \ldots \ldots$ & 0,0007 & 0 & 0 \\
\hline Groupe i2 (4 poulets) & $\ldots \ldots \ldots \ldots \ldots$ & 0,0005 & o & o \\
\hline
\end{tabular}

montre que deux antioxygènes sont capables de s'accumuler dans les graisses de ces oiseaux : le DPPD et le BHT.

Toutefois, le taux de DPPD retrouvé chez le poulet (o,0007 p. roo) est inférieur à celui qui a été trouvé chez le porc. Les concentrations sont deux à trois fois plus faibles dans le premier cas que dans le second. Ces résultats ont été confirmés par le fait que les graisses des oiseaux traités ne sont pas stabilisées, alors que la période d'induction est notablement accrue dans le cas du porc.

En revanche, le BH'T (ou DBPC) qu'il n'avait pas été possible de mettre en évidence dans les graisses du porc, existe ici à une concentration aisément dosable (o,006 p. Ioo). Il s'agit bien de BHT ou de corps voisins, car la graisse d'animaux témoins ne donnait pas la réaction colorée du BH'T. Fnfin, comme dans le cas de DPPD chez le porc, ces dosages ont été confirmés par l'étude de la stabilité des graisses de réserve. Ia période d'induction des grajsses des animaux ayant reçu du BHT était notablement augmentée.

\section{Influence de l'ingestion d'antioxygènes sur la stabilité des graisses vis-à-vis de l'oxydation}

I a stabilité des graisses vis-à-vis de l'oxydation est fonction de deux facteurs principaux qui agissent en sens opposé : d'une part le taux d'acides gras insaturés et, d'autre part le taux d'antioxygènes.

Pour ce qui concerne le premier de ces facteurs, nous avons déterminé sur chacun des échantillons l'indice d'iode qui, en première approximation, donne une mesure de la quantité d'acides gras insaturés présents dans la graisse.

I.es indices d'iode moyens des graisses de porc étudiées sont résumés dans le tableau $\mathrm{V}$.

Les indices ne sont pas notablement modifiés dans la plupart des lots. En conséquence, l'addition d'antioxygènes aux rations ne risque donc pas de modifier considérablement le point de fusion des graisses. 


\section{TABLEAU $\mathrm{V}$}

Indice d'iode des tissus adipeux du porc

\begin{tabular}{|c|c|c|c|}
\hline & Lard externe & Lard interne & Panne \\
\hline 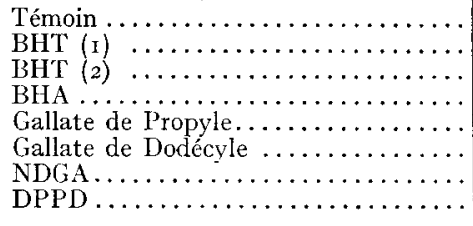 & $\begin{array}{l}62,3 \\
62,5 \\
64,2 \\
62,7 \\
61,4 \\
65,2 \\
62,8 \\
64,7\end{array}$ & $\begin{array}{l}55,5 \\
56,9 \\
58,7 \\
56,6 \\
55,5 \\
61,1 \\
57,0 \\
57,3\end{array}$ & $\begin{array}{l}48,4 \\
47,5 \\
49,4 \\
49,6 \\
49,3 \\
51,8 \\
49,2 \\
47,7\end{array}$ \\
\hline
\end{tabular}

Toutefois, il existe une différence significative entre l'indice d'iode moyen du lot témoin et celui du lot DPPD. I1 y aurait donc "épargne" des acides gras insaturés chez les animaux ayant ingéré l'antioxygène. Cette observation a déjà été faite par BRATZLER et al. (I950) qui étudiaient la composition des graisses de pores. Chez les animaux recevant des tocophérols, il y avait accumulation d'acide oléique aux dépens des acides gras saturés. Toutefois, GaR'ron et al. (I958) n'ont pas confirmé ces résultats, et Johnson, O'Halloran et HEGWill (1958) ont constaté une influence variable selon le taux de BHA contenu dans la ration.

Pour les antioxygènes, nous avons effectué le dosage direct dont les résultats sont mentionnés dans les tableaux I à IV. En outre, nous avons utilisé une méthode indirecte qui consiste à étudier la stabilité des graisses vis-à-vis d'une oxydation accélérée artificiellement. On étudie ainsi la cinétique de l'évolution du taux des péroxydes des graisses Ces échantillons qui ont été prélevés sur des animaux ayant reçu des antioxygènes sont comparés d'une part aux graisses d'animaux témoins (sans antioxygènes) et, d'autre part à des échantillons de graisse de porcs témoins, additionnés de quantités connues de chacun des antioxygènes. Par comparaison de la durée des périodes d'induction, il est ainsi possible de déterminer l'ordre de grandeur de la teneur en antioxygènes du tissu gras.

Porc.

La figure I montre l'évolution de l'indice de péroxydes des graisses maintenues à $60^{\circ} \mathrm{C}$. Nous avons calculé la moyenne des durées (en heures) nécessaires pour atteindre des indices de péroxydes éganx à 5 , Io et 20 . Cette courbe concerne le lard interne.

Par ailleurs, les figures 2 et 3 indiquent l'évolution de graisses témoins enrichies de quantités données d'antioxygènes : o,O0I p. I00 et o,O00I p. I00.

La figure I (I) montre très clairement que le DPPD ingéré par l'animal stabilise considérablement les graisses de réserve vis-à-vis de 


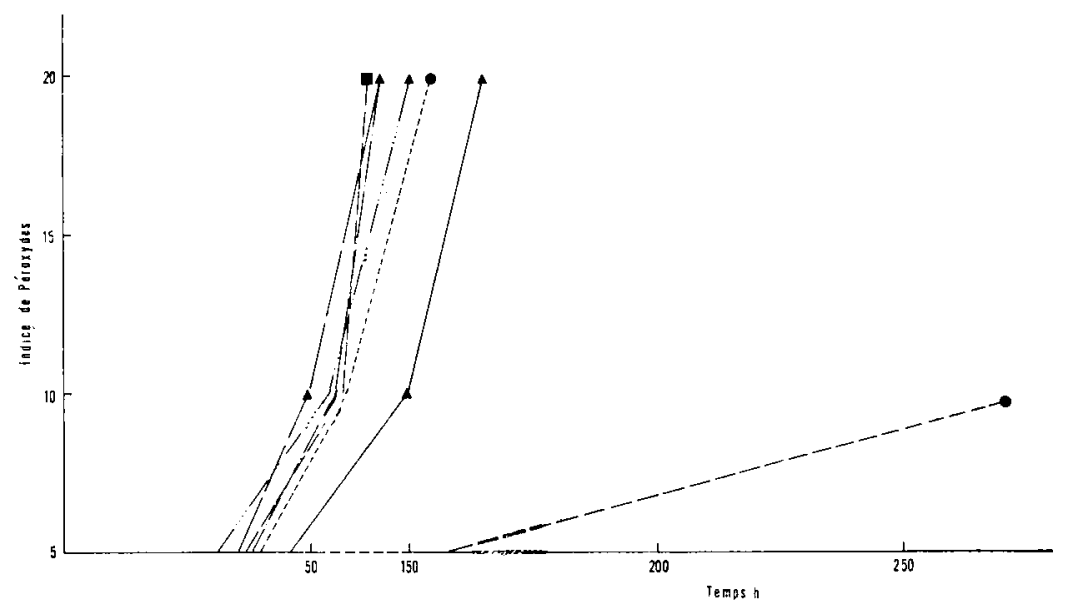

Fig. I. - Évolution de l'indice de péroxydes d'échantillons de lard interne de porc (animaux ayant ingéré les différents antioxygènes).

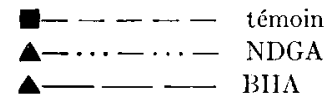

A- - - - - Gallate de dodecyle

........... Gallate de opylpre

A- BHT

- - DPPD

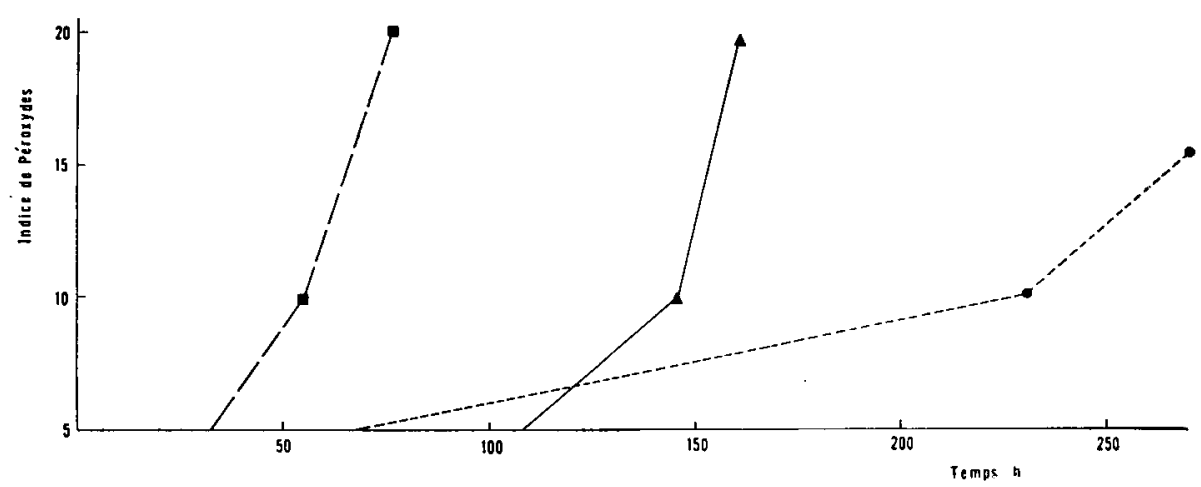

FIG. 2. - Évolution de l'indice de péroxydes de lard interne de porc additionné de o,oor p. roo de DPPD ou de BHT.

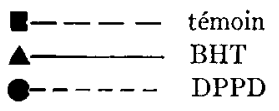


l'oxydation. Par ailleurs, la figure 2 montre que l'addition de $0,00 \mathrm{I}$ p. Ioo de DPPD permet d'atteindre un indice de péroxydes égal à ro en 200 heures environ; la valeur 20 est atteinte en 300 heures. Or, c'est l'ordre de grandeur des valeurs relevées sur la figure I. On peut en conclure que l'ordre de grandeur de la concentration en DPPI) est o,oor p.IOo, alors que le dosage direct aboutit à un taux compris entre 0,00 I2 et $0,0025 \mathrm{p}$. Ioo. L'étude de la cinétique du taux de péroxydes confirme donc le résultat du dosage direct.

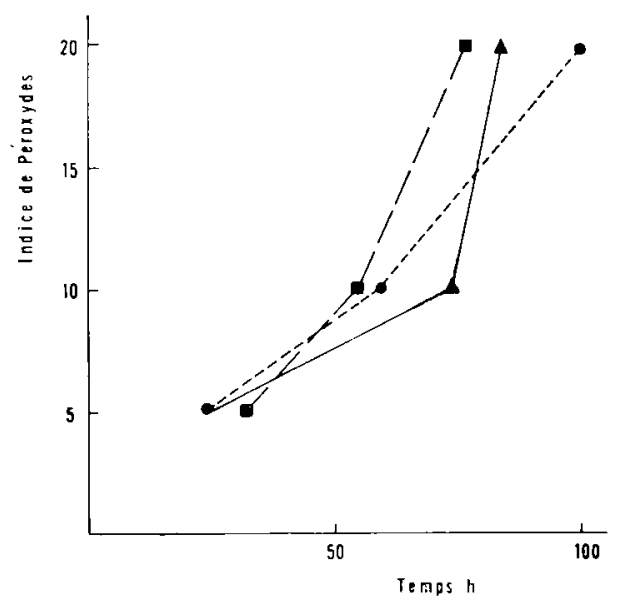

FIG. 3 - Evolution de l'indice de péroxycles de lard interne de porc additionné de o,ooor p. I00 de DPPD ou de BSITT.

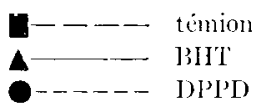

I es lards internes du lot ayant reçu le BH'T semblent légèrement stabilisés par rapport aux autres lots. L'étude de la figure 2 montre également que le BH'T ajouté atux graisses à la dose de o,OOI p. Ioo conduit à l'apparition d'un indjce de péroxydes égal à 20 après environ I 60 heures. Toutefois, Ia comparaison des figures I et 3 permet de conclure que le taux d'antioxygènes éventuellement stocké dans la graisse serait inférieur à $0,00 \mathrm{I}$ p. IOO, et vraisemblablement voisin de $0,000 \mathrm{I} p$. IOo (indice 20 après 80 heures). En conséquence, on peut présumer un léger passage de BH'T, mais en quantité trop faible pour qu'il puisse être mis en évidence au moyen d'un dosage chimique.

Pour les autres antioxygènes, il est impossible de mettre en évidence une stabilisation significative des graisses. Le gallate de propyle, toutefois, accroît la période d'induction, bien qu'il soit inpossible de le mettre en évidence par dosage. Ceci peut être dû à la sensibilité du dosage, ou bien à un effet indirect; en effet, l'antioxygène de synthèse ajouté au régime pourrait provoquer une "épargne " d'antioxygènes naturels tels 
que les tocophérols. 'Toutefois, dans la plupart des cas, le dosage direct dans le tissu permet d'écarter cette hypothèse. Dans le cas du gallate de propyle, il pourrait s'agir de la présence d'un produit de transformation de cette substance, différent de l'acide gallique, puisque la réaction avec $\mathrm{FeCl}_{3}$ était négative.

Poulet.

I'indice d'iode des graisses de poulet est notablement plus élevé (67 à 72) que celui des graisses de porc. Par ailleurs, le taux du DPPD est 3 à 4 fois plus faible que celui qui correspond aux graisses de porc. Or, ces deux caractéristiques modifient l'efficacité de l'antioxygène. En effet, les graisses des poulets du lot DIPD ne sont pratiquement pas stabilisées. En revanche, le BHT, que nous avons par ailleurs dosé au taux de 0,006 p. Ioo, stabilise considérablement les graisses du poulet (fig. 4). I,es deux méthodes, directe et indirecte, donnent ainsi par recoupement un renseignement comparable.

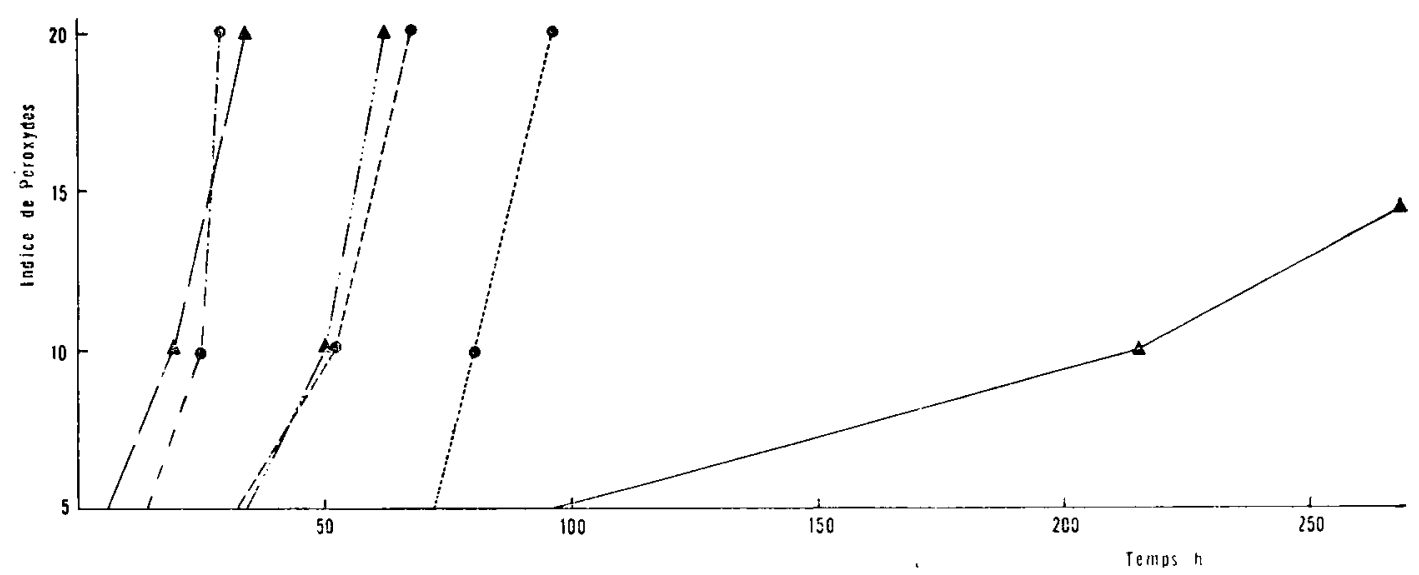

Fic. 4. - Évolution de l'indice de péroxydes d'úhantillons de graisse de poulets (animaux ayant ingéré les différents antioxygènes).

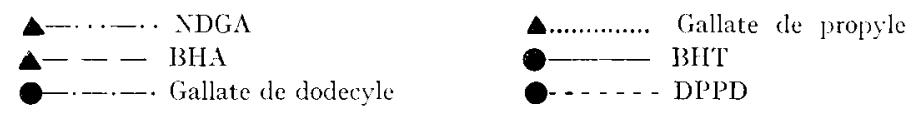

\section{DISCUSSION}

Les résultats obtenus au cours de ces recherches ont ainsi permis de mettre en évidence le passage de certains antioxygènes dans les productions animales. Ce passage concerne essentiellement le tissu gras dans lequel, chez le porc, le DPPD est seul pratiquement capable de se stocker. Chez le poulet, le DPPD est également capable de franchir la barrière intestinale et de s'accumuler dans les graisses. Il en est de même pour le BHT, mais la concentration de cet antioxygène atteint 
des valeurs très supérieures à celles qui correspondent au DPPD. I,es taux trouvés sont toutefois faibles en valeur absolue : 1'ordre de grandeur est au maximum de 0,005 p. Ioo.

Il convient également de souligner que le taux de 0,1 ou de 0,125 p. Ioo d'antioxygène incorporé aux régimes expérimentaux est dix fois plus élevé que celui qui est recommandé pour stabiliser les graisses. Toutefois, la stabilisation de certains aliments (farines animales notamment) peut exiger des concentrations élevées d'antioxygènes. De même, le taux d'incorporation d'antioxygènes aux aliments destinés aux volailles, afin de prévenir l'encéphalomalacie peut être relativement élevé. Néanmoins, les concentrations que nous avons utilisées dans ces régimes constituent un maximum qui ne serait pas atteint dans la pratique. En conséquence, on peut présumer que les taux retrouvés dans les tissus seraient notablement plus faibles que ceux que nous avons observés.

Néanmoins, l'utilisation des antioxygènes dans l'alimentation des animaux pose un problème qui présente un double aspect. Du point de vue de la technologie des produits animaux, l'accumulation de certains antioxygènes dans les tissus, et notamment dans les tissus gras, constitue un facteur favorable puisqu'elle a pour corollaire une meilleure résistance à l'oxydation de ces graisses. Toutefois, pour l'hygiéniste, il est indispensable d'exiger que l'antioxygène présent dans les tissus ne présente aucun inconvénient pour le consommateur de viande de porc ou de poulet. Or, on connaît maintenant la toxicité du DPPD (Ames et al.. 1956) ; OSER et OSER (I956) ont montré que cet antioxygène provoque un accroissement de la durée de la gestation chez la rate, et qu'il en résulte une forte mortalité chez les mères et les jeunes. In revanche, il paraît bien établi que le BHT est un corps très peu toxique (DEICHMAN et a1., I955).

Compte tenu de la stabilité soit du DPPD, soit du BH'T, vis-à-vis des agents chimiques, il y a de fortes présomptions pourque la moléctle de ces corps n'ait pas été modifiée au cours de l'absorption intestinale, et du transport.

Par ailleurs, la bonne concordance qui existe entre le dosage direct des an tioxygènes et la période d'induction des graisses permet de renforcer cette opinion. Toutefois, le problème des produits de transformation des produits ingérés ne doit pas être minimisé. Johnson, O'HALI,ORANet HEGWILL (I 959) ont souligné les difficultés qui s'attachent à l'identification de ces produits.

\section{SUMMARY}

The influence of the ingestion of various antioxidants on their final accumulation in muscle, reserve fats (dorsal and perinephric), liver and kidney, and on the resistance to oxidation of the reserve fats, has been studied in the pig and the chicken.

The animals received in the ration either o,I25 p. Ioo diphenyl-p. pheny- 
lenediamine (DPPD) or o,I p. Ioo butylated hydroxyanisole (B. H. A.), butylated hydroxytoluene (BHT), dodecyl gallate, propyl gallate, of nordihydroguaiaretic acid (NDGA).

The experiment lasted four months for the pig and eight weeks for the chicken. The BHA, NDGA and gallates were extracted with petroleum ether and reextracted with $72 \mathrm{p}$. Ioo alcohol. They were determined by means of the $\mathrm{FeCl}_{3}$-dipyridyl reaction (KAHAN, I954). The $\mathrm{BH}$ 'T was distilled over with superheated steam, at $\mathrm{r} 8 \mathrm{o}^{\circ} \mathrm{C}$ (modification of the method of ANGLIN, MAHON, ChAPMAN, 1956) and also determined colorimetrically. The DPPI was extracted after saponification of the fatty tissue and its nitrated derivative determined by colorimetry. (BUNNEI, I954).

In the pig, only DPPD is detectable in the reserve fats (maximum amount found : 0,0025 p. I00,. (Tables I and II), and in the kidney (maximum 0,0006 p. IOo). In the chicken, DPPI) also accumulates in the reserve fats, but the concentration is lower $(0,0007 \mathrm{p}$. IOo) than in the fat of the pig (Table III). On the other hand, BHT is stored in the fat of the chicken at a level of $0,006 \mathrm{p}$. Ioo.

The study of the stability of reserve fats (development of the peroxide index, in an oven regulated at $60^{\circ} \mathrm{C}$ and illuminated) of control and treated animals confirms the result of direct determination of the antioxidants. Indeed, the fats of pigs which had ingested DPPD (fig. I) and those of chickens which had received BHT (fig. IV) show an induction period which is much longer than that of the fats of control animals. However, one observes induction periods of the same order of magnitude on the addition of known quantities of $\mathrm{BHT}$ or DPPD (o,oor and $0,000 \mathrm{I}$ p. roo) to the fats of control pigs (fig. 2 and 3 ).

The technological and hygienic consequences of these observations are discussed.

\section{REFERENCES BIBLIOGRAPHIQUES}

Ames S. R., Ludwig M. I., Swanson W. J. and Harris P. L., I956. Effect of DPPD, methylene blue, BHT, and hydroquinone on reproductive process in the rat. Proc. Soc. Exp. Biol. Med., 93, 39.

Anglin C., Mahon J. H. and Chapman R. A., I956. Determination of antioxidants in edible fats. Agric. Food Chem., 4, ror8.

Barnes R. H., Lundieng W. O., Hanson H. T. and Burr G. O., I943. The effect of certain dietary ingredients on the keeping quality of body fat. J. Biol. Chem., 149, 3 I 3 .

Bratzler J. W., Loosli J. K., Krukovski V. N. and Maynard L. A., I950. Effects of the dietary level of tocopherol on their metabolism in swine. J. Nutr., 42, 59 .

Bunner, R. H., r954. The determination of diphenyl-p-phenylenediamine (DPPD) in feeds. Progress report $7-\mathrm{July}$, Department of Poultry Science Storrs Agricultural Experiment Station - Connecticut.

Burr G. O., LundberG W.O. and Chipault J. R., I946. The role of various substances in stabilizing animal tissues. Oil and Soap, 23, 382 .

Carpenter L. E. and LundBerg W. O., I949. Effect of tocopherols on vitality of pigs in relation to "baby pig disease ". Ann. N. Y. Acad. Sci., $52,269$.

Chipault J. R., Lundberg W. O. and Burr G. O., I945. The chemical determination of tocopherols in animal fats : the stability of hog fats in relation to fatty acid. Composition and tocopherol contents. Arch. Biochem., 8, $32 \mathrm{r}$. 
Criddre J. E., and Morgan A. F., I95I. Effect of tocopherol feeding on the composition of turkey tissues. Proc. Soc. Exp. Biol. Med., 78, 4I.

Deichman W. B., Cremmer J. J., Rakoczy R. and Bianchine J., I955. Toxicity of ditertiarbutylmethylphenol. Arch. Ind. Health, 11, 93.

Garion G. A., Duncan W. R. H., Marsden K. A., Shanks P. L. and BEATTLE I. S., I958. Observations on feeding pigs on a low-fat diet with and without supplementary tocopherol. Brit. J. Nutr., 12, 97.

HANLEY J. W., I953. Antioxidant treatment of bacon. Food Techn., \%, 429. Hanson H. T., Barnes R. H., I,Undberg W. O. and Burr G. O., I944. The deposition of antioxidants in the abdominal fat depots. J. Biol. Chem., 156, 673.

Hoom M. P., WHEeler R. S. and MCGlamery J. B., I950. Oxydative changes in estrogen stimulated fat and the influence of natural tocopherol on stability of fats in normal chickens. Poult. Sci., 29, 824 .

Johnson A. R., O'Hallorax M. W. and Hewgill, F. R., I959. Phenolic antioxidants and the stability of perirenal rat fat. J. Amer. Oil Chem. Soc., 35, 496 .

KAHAN S., I954. Determination of nordihydroguaiaretic acid and butylated hydroxyanisol. J. Ass. off. agric. Chem. Wash., 3\%, 828 .

Lundberg W. O., Barnes R. H., Clausen M. and Burr G. O., I944. The deposition and storage of $\alpha$-tocopherol in abdominal fats. J. Biol. Chem., 153,265 .

Lundbekg W. O., Barnes R. H., Ciausen M., Larson N. and Burr G. O., I947. The deposition and antioxygenic behavior of $\alpha, \beta$, and $\gamma$-tocopherols in rat fats. J. Biol. Chem., 168, 379 .

Lynch G. P., Anderson G. C. and Liwis W. R., I958. Stabilization of hog carcass fats by the addition of antioxidants to the ration. J. Anim. Sci., 1\%, II5I.

Major R. and WaT'Ts B. M., I948. The relation of fed and injected tocopherols to development of rancidity in the stored meat and ritilization of carotene by the rabbit. J. Nutr., 35, 103.

Mecchi E. P., Pool M. F. and KLOSE A. A., I953. The role of tocopherol content in the stability of chicken and turkey fats. Poult. Sci., 32, 9I5.

Mecchi E. P., Pool M. F., Behman G. A., Hamachi M. and Klose A. A., I $956 \mathrm{a}$. The role of tocopherol content in the comparative stability of chicken and turkey fat. Poult. Sci., 35, I238.

Mecchi E. P., POOL M. F., Nonaka N., Klose A. A., Marsden S. J., LIILIE R. J., I956 b. Further studies on tocopherol content and stability of carcass fat of chickens and turkeys. Poult. Sci., 35, I246.

OSER B. I. and OSER M., 1956. Inhibitory effect of feed grade diphenyl-pphenylenediamine (DPPD) on parturition in rats. J. Agric. Food Chem., 4, 796 .

Pudelikienicz W., Potter L. M., Matterson L. D. and Singsen E. P., I956. A semi quantitative method for the determination of DPPD in chicken fat and eggs. Poult. Sci., 35, 959.

Watts B. M., CunHa T. J. and Major R., I946. Effect of feeding and injecting hogs with tocopherols on the susceptibility of pork fat to rancidity. Oil and Soap, 23, 254.

WENGER von F., I954. Antioxydantien in Fetten und Olen. Einfache Nachweismethoden für Antioxydantien. Mitt. Gebensm. Hyg., Bern, 45, 383 . 